\section{WEB WATCH}

A bit of micro magic

- http://pga.tigr.org/tigrscripts/magic/r1.pl

Microarrays have

revolutionized biology, so it is little wonder that much

attention has been devoted

to their design and analysis.

But if it is difficult to analyse

single microarray

experiments, what about

cross-experiment

comparisons? TIGR has

risen to the challenge of

cross-referencing mouse, rat

and human microarray

expression data in the

RESOURCERER database.

Given that these rodents are important models of human development and disease, such comparisons are valuable for studies of human gene function.

The RESOURCERER is a

database that allows you to do pairwise comparisons between 43 expression microarray resources (20 from mouse, 15 from human and 8 from rat) that have been generated on various platforms (such as cDNA or oligonucleotide arrays) by both academic and commercial labs. For example, you can compare a mouse cDNA data set to one generated from a mouse oligonucleotide array or from a human $U 95$ GeneChip ${ }^{\text {TM }}$. You can choose to view information that is shared between data sets or that is unique to one, or the other. The output of such comparisons is a table that provides information on each array element. This information includes GenBank and LocusLink identifiers, links to mammalian orthologues, and to Gene Ontology terms based on TIGR annotations. Finally, you can also search the database using GenBank accession numbers.

RESOURCERER harbours a wealth of information and has the potential to contain further data sets. Even if you are not a computational analysis aficionado it is worth a visit - the web site is very clear, with step-by-step instructions.

Magdalena Skipper

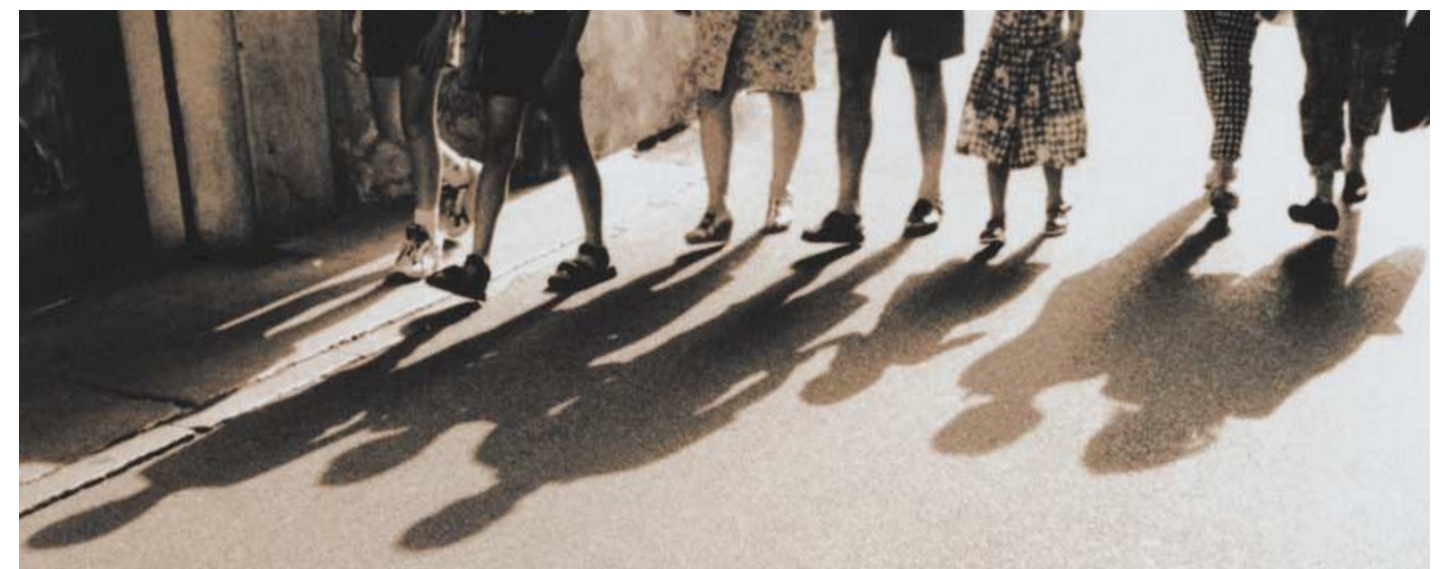

DEVELOPMENTAL BIOLOGY

\title{
Limbs make progress
}

According to the well-established progress zone (PZ) model of vertebrate limb development, undifferentiated cells acquire positional information in the $\mathrm{PZ}$ - a region at the distal end of the developing limb. The fate of these cells is set by how long they spend in this zone: cells that leave early contribute to proximal limb structures and cells that leave late, to distal ones. This theory neatly explains why removing the apical ectodermal ridge (AER) — which overlies the $\mathrm{PZ}$ and is a likely source of signalling molecules, such as the Fgfs (fibroblast growth factors), that are involved in distal limb patterning — from developing limbs causes the truncation of distal structures. Now, two Nature papers question the validity of this model and shed new light on the cellular events that occur during limb development.

Gail Martin's group took a genetic approach to investigating limb development by conditionally inactivating both $F g f 4$ and $F g f 8$ in the mouse limb. Although the hindlimbs of this mutant do not form, their forelimbs do, owing to the early transient expression of Fgf8 and Fgf4. These limbs have normal proximal structures, as predicted by the $\mathrm{PZ}$ model, and more distal elements. However, they often lack middle limb structures - a phenotype that the PZ model, which requires that distal elements are specified only after more proximal ones, cannot easily explain. Sun et al. suggest that the poor development of mutant forelimb skeletal structures is due, not to a lack of progressive specification, but to the loss of skeletal precursor cells, as assayed by Sox 9 expression, caused by abnormal cell death in the most-proximal part of mutant limb buds.

Cell death also features in the study by Cliff Tabin's group. Dudley et al. found that, on AER removal, a region of cell death would extend $200 \mu \mathrm{m}$ from the bud's distal tip. Could this cell death alone account for the distal limb truncations that occur on AER ablation? If this celldeath zone remained constant in size over several developmental stages, as indicated by the authors' studies, then a proportionally larger part of the distal limb would be lost at each stage.

According to the $\mathrm{PZ}$ model, on AER removal, distal cells should incorporate into proximal populations as their distal fate should not yet be fully specified. Instead, Dudley et al. found that distal cells die, while proximal cells expand and develop. Together these data indicate that patterning after AER loss might reflect the proportion of cells that lie outside the AER's influence - and that therefore remain unaffected by the changes in cell death and proliferation caused by its removal — rather than the level of patterning achieved by a particular developmental stage, as predicted by the PZ model.

In subsequent cell-fate mapping and embryological studies, the authors found evidence to indicate that positional specification and fate committment might be two distinct events in limb development. By labelling proximal, middle and distal cell populations, they found that cells in these regions do not intermix, indicating that they might be positionally specified at an early stage. Grafting experiments supported this conclusion, and additional cell reaggregation studies indicated that the fate of distal cells is not fixed until later in limb development.

The new picture of limb development proposed by Tabin's group is one in which cell fates are established early, rather than progressively, and are then fixed in a proximal-distal wave of precursor-cell expansion and differentiation. Martin's group report limb phenotypes that cannot readily be explained by the PZ model, and provide new evidence on the involvement of Fgfs in preventing cell death, in influencing the initial size of the limb bud, and in maintaining cell proliferation and gene-expression programmes during limb development. Although dispensing with the PZ model might be premature, further progress in the field might yet lead to its downfall.

Jane Alfred

6) References and links ORIGINAL RESEARCH PAPERS Sun, $X$. et $a l$. Functions of FGF signalling from the apical ectodermal ridge in limb development. Nature 418, 501-508 (2002) | Dudley, A. T. et al. A re-examination of proximodistal patterning during vertebrate limb development. Nature $\mathbf{4 1 8}$ 539-544 (2002) 